\title{
Non-Determinism and Nash Equilibria for Sequential Game over Partial Order
}

\author{
Stéphane Le Roux ${ }^{12 \dagger}$ \\ ${ }^{1}$ LIP, Ecole normale supérieure de Lyon, France. http://perso.ens-lyon.fr/stephane.le.roux/ \\ ${ }^{2}$ JAIST, Nomi, Japan
}

received February 2006, revised $26^{\text {th }}$ June 2006, accepted tomorrow.

In sequential games of traditional game theory, backward induction guarantees existence of Nash equilibrium by yielding a sub-game perfect equilibrium. But if payoffs range over a partially ordered set instead of the reals, then the backward induction predicate does no longer imply the Nash equilibrium predicate. Non-determinism is a solution: a suitable non-deterministic backward induction function returns a non-deterministic strategy profile which is a nondeterministic Nash equilibrium. The main notions and results in this article are constructive, conceptually simple and formalised in the proof assistant Coq.

Keywords: Nash equilibrium, sequential game, non-determinism, partial order, constructive, proof assistant

\section{Introduction}

Neumann and Morgenstern (1944) described the basis of modern game theory. Then Nash (1950) introduced his key notion of non-cooperative equilibrium. That concept referred to strategic games in the first place, but is also relevant when studying games in extensive form, also called sequential games. An early equilibrium existence proof is due to Zermelo (1912) for the game of Chess, which is a specific instance of sequential game. Kuhn (1953) stated the existence of equilibrium for games in extensive form in 1953. Vestergaard (2006) formalised this result with the theorem prover Coq, for binary game trees and payoffs ranging over the naturals. The proof relies on a notion called backward induction in game theory and introduced by Selten (1965) and Selten (1975). Game theory has been mostly studying real-valued payoffs games, although Blackwell (1956) generalised strategic games by replacing real-valued payoffs by real-valued payoffs vectors. As a result, strategy profiles were no longer preference-wise totally ordered. That lead to the definition and study of multi criteria games.

\subsection{Issues and Contribution}

As is clear from Vestergaard (2006), Kuhn's argument relies on the domain of payoffs to be totally ordered. The issue addressed here is what happens to Kuhn's result and its formal proof when payoffs range over

\footnotetext{
${ }^{\dagger}$ This research was partially supported by CDFJ (Collège doctoral franco-japonais) 
an arbitrary partial order. Note that considering partial orders is both an abstraction and a generalisation over real-valued payoffs (vectors). An example shows that not all games have Nash equilibria under the definitions of strategy and Nash equilibrium directly translated from the real-valued payoffs case. Those classic definitions can be refined along Le Roux et al. (2006) so that there seems to be no simple counter example to equilibrium existence. However an example shows that backward induction no longer implies Nash equilibrium (which does not mean that there is no Nash equilibrium). It means that an equilibrium-existence proof in the style of Vestergaard (2006) is not possible. I introduce the formal notions of non-deterministic strategy and non-deterministic equilibrium, and define a non-deterministic backward induction function. Then I prove constructively and formally with Coq that any sequential game with partially ordered payoffs has a non-deterministic equilibrium.

\subsection{This Article}

Section 2 gives an overview of what would be the formalism from Vestergaard (2006) used with an arbitrary partial order. Section 3 shows that the definitions of Nash equilibrium and backward induction need slight rephrasing at the logical level (as done in part by Le Roux et al. (2006)), and that the backward induction predicate no longer guarantees the Nash equilibrium predicate. Section 4 formally introduces, in the non-deterministic case, the notions of strategy and Nash equilibrium, and defines a backward induction function. The end of the section states and sketches the proof of equilibrium existence for sequential games when payoffs range over an arbitrary partial order.

The content of this article is formalised in Coq. For the sake of simplicity only binary game trees are involved. However, the result should hold for all games in extensive form. I mainly follow Vestergaard (2006) and Le Roux et al. (2006) for notations and terminology.

\section{Basic Definitions}

Informally speaking, a game involves some players, also called agents. For each agent the set of possible payoffs, also called outcomes, is (partially) ordered by a preference relation. A game in extensive form is represented as a rooted tree with labelled nodes: internal nodes are labelled with an agent, and leaves with a payoff function. A payoff function takes an agent and returns a payoff. The game starts at the root. The agent owning the root chooses in which sub-tree the game will continue, and so on. When the play reaches a leaf, payoffs are distributed and the game ends. Formally, games are defined by induction:

Definition 1 ((Binary) Game Trees, Payoff Function) Let $\mathcal{A}$ be a set of agents and let Payoffs be a set of payoffs with a decidable order relation.

$$
\mathrm{G}::=\underset{\text { gN } P F}{\mathcal{A} G \mathrm{G}} \quad \mathrm{PF}: \mathcal{A} \rightarrow \text { Payoffs }
$$

An example involving two agents $a$ and $b$

$$
\operatorname{gN} a(\mathbf{g N} b(a \mapsto 1, b \mapsto 0)(a \mapsto 3, b \mapsto 1))(a \mapsto 2, b \mapsto 2)
$$

and its graphical representation. The label $A, B$ at a leaf means that $a$ gets payoff $A$ and $b$ gets payoff $B$.

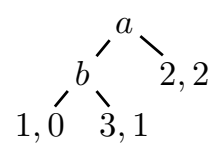


In order to play, agents follow strategies. Informally, a strategy for one agent amounts to a choice being (pre-)made at each internal node owned by the agent. A strategy profile is a tuple of strategies, one strategy per agent. A strategy profile amounts to a choice being (pre-)made at each internal node of the game. Formally, choices and strategies are defined by induction:

\section{Definition 2 ((Binary) Strategy profiles, Choices)}

$$
\begin{aligned}
\mathrm{S}::= & \mathrm{sL} \mathrm{PF} \\
\mid & \mathbf{s N} \mathcal{A} \text { Choice S S }
\end{aligned}
$$

We let c range over Choice and write $\overline{\mathrm{c}}$ for c- "opposite”: $\overline{\mathrm{l}} \triangleq \mathbf{r}, \overline{\mathbf{r}} \triangleq \mathbf{l}$.

Informally, the payoff function induced by a strategy profile is at the leaf that is reached by the play following the "recommendation" of the strategy profile. Formally the induced payoff function is defined by induction:

\section{Definition 3 (Induced Payoff)}

$$
\begin{gathered}
\mathrm{PO}(\mathbf{s} \mathbf{L} \text { po }) \triangleq p o \\
\mathrm{PO}\left(\mathbf{s N} a \mathbf{c s}_{\mathbf{l}} \mathbf{s}_{\mathbf{r}}\right) \triangleq \mathrm{PO}\left(\mathrm{s}_{\mathrm{c}}\right)
\end{gathered}
$$

An Example of strategy profile using the previous game

$$
\mathbf{s N} a \mathbf{l}(\mathbf{s N} b \mathbf{l}(a \mapsto 1, b \mapsto 0)(a \mapsto 3, b \mapsto 1))(a \mapsto 2, b \mapsto 2)
$$

and its graphical representation. Bold lines between nodes represent choices (pre-)made by the agents. The induced payoff function of that strategy profile is: $a$ gets 1 and $b$ gets 0 .

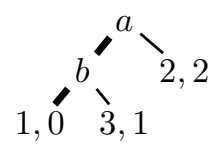

Intuitively, when a strategy profile is given, an agent owning some nodes can change his strategy, e.g., go left instead of right in any node he owns. This leads to a change in the strategy profile. That is formally captured by the following definition:

\section{Definition 4 (Sequential-Game Convertibility)}

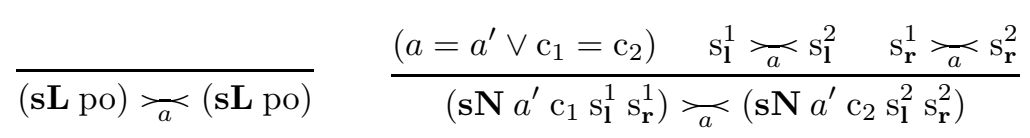

For example, below, the agent $b$ can convert the strategy profile on the left to the one on the right.
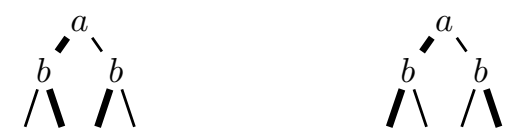

Informally, a Nash equilibrium is a strategy profile that no agent can convert to another strategy profile strictly better for himself. Formally it is defined below, but not by induction: 


\section{Definition 5 ((Sequential) Nash Equilibrium)}

$$
\mathrm{Eq}^{\mathrm{N}}(\mathrm{s}) \triangleq \mathrm{s}^{\prime}, a \cdot \mathrm{s} \underset{a}{\prec} \mathrm{s}^{\prime} "=>\text { PO }\left(\mathrm{s}^{\prime}\right)(a) \leq \mathrm{PO}(\mathrm{s})(a)
$$

Backward induction is a predicate. Intuitively, it amounts to optimising choices (pre-)made at each internal node of the game, when the options are comparable. Formally, it is defined by induction:

\section{Definition 6 (Backward Induction)}

$$
\overline{\mathrm{BI}(\mathbf{s L} \text { po })} \quad \frac{\mathrm{BI}\left(\mathrm{s}_{\mathbf{l}}\right) \mathrm{BI}\left(\mathrm{s}_{\mathbf{r}}\right) \mathrm{PO}\left(\mathrm{s}_{\overline{\mathrm{c}}}\right)(a) \leq \mathrm{PO}\left(\mathrm{s}_{\mathrm{c}}\right)(a)}{\mathrm{BI}\left(\mathbf{s N ~ a ~ c ~ s} \mathrm{s}_{\mathbf{l}}\right)}
$$

Not all Nash equilibria are backward induction. For example the underlying game below has two Nash equilibria. Only the one to the left is backward induction but both are Nash equilibria.

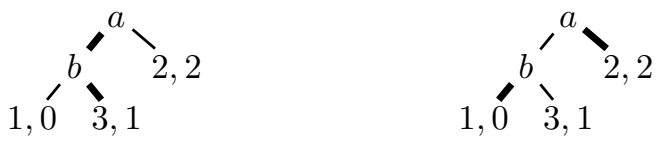

Note that in the total-order case, backward induction implies Nash equilibrium, as proved by Vestergaard (2006). However, Subsection 3.2 shows that this does not hold in the partial-order case.

\section{Problems}

\subsection{First Problem}

According to the current definitions of equilibrium and backward induction, not all games (in the partial order case) have equilibria and the backward induction predicate is empty. This happens in the following example where the agent $a$ plays alone and $x$ and $y$ are two outcomes that are not comparable. Indeed, none of the two possible strategies would be explicitly better than or even equivalent to the other one.

$$
\stackrel{a}{x}
$$

The above "problem" (one usually expects that equilibrium exists) has a solution: the intuition behind the notion of equilibrium is that an agent cannot get something explicitly better by changing his mind, not that what he would get is explicitly worse or equal (for an discussion on this topic, see Le Roux et al. (2006)). The two concepts lead to equivalent predicates for total orders but not for partial orders. Hence the two definitions of equilibrium and backward induction can be slightly changed to:

$$
\begin{aligned}
& \mathrm{Eq}^{\mathrm{N}}(\mathrm{s}) \triangleq \mathrm{s}^{\prime}, a \cdot \mathrm{s} \underset{a}{\prec} \mathrm{s}^{\prime} "=>" \neg\left(\mathrm{PO}(\mathrm{s})(a)<\mathrm{PO}\left(\mathrm{s}^{\prime}\right)(a)\right) \\
& \overline{\mathrm{BI}(\mathbf{s L} \text { po })} \quad \frac{\mathrm{BI}\left(\mathrm{s}_{\mathbf{l}}\right) \mathrm{BI}\left(\mathrm{s}_{\mathbf{r}}\right) \neg\left(\mathrm{PO}\left(\mathrm{s}_{\mathrm{c}}\right)(a)<\mathrm{PO}\left(\mathrm{s}_{\overline{\mathrm{c}}}\right)(a)\right)}{\mathrm{BI}\left(\mathbf{s N ~ a ~ c ~} \mathrm{s}_{\mathbf{l}} \mathrm{s}_{\mathbf{r}}\right)}
\end{aligned}
$$

The re-definitions above can actually be seen as a refinement of the two notions. Now it would be possible to guarantee that the backward induction predicate is not empty. However the question would be: Is the proof of Vestergaard (2006) still a proof for the existence of equilibrium in the partial-order case? Put otherwise, does the backward induction predicate still guarantee the Nash equilibrium predicate? 


\subsection{Second Problem}

The answer to the last question above is negative due to the following example where the agent $a$ plays alone and the only comparison between $x, y$, and $z$ is $z \leq x$. The strategy profile below is a backward induction but the agent can get something strictly better by shifting his two choices to the left.

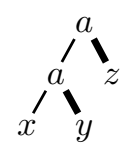

It implies that although an equilibrium may exist, the proof cannot have the same structure as for the total-order case. There are two main ways to continue: either change proofs, or change definitions. For now, let see why it is reasonable to consider changing the definitions so that the proof does not need to change to much in spirit. Nash encountered the same kind of problem when dealing with strategic games: not all of those games had an equilibrium. So Nash (1950) introduced probabilities into the definitions of strategy and equilibrium, so that he could guarantee existence of (probabilistic) equilibrium. The essence of Nash's construction is explained by Le Roux et al. (2006). Similarly, the Nash-like construction of Section 4 will cope with the partial order issue.

\section{Non-Deterministic Strategies}

According to the current definition, a strategy profile amounts to exactly one sub-game tree being chosen at each node of the game tree. The underlying strategies are therefore implicitly deterministic. The notion of non-deterministic strategy will generalise the one of strategy and guarantee equilibrium existence. The non-determinism used here is explicit and discrete. The discreteness guarantees that finite games generate finite sets of strategies, which has a computational advantage. As for Nash's probability construction for finite strategic games, the non-determinism is induced by probabilities and is therefore "continuous". So, in Nash's case, finite games generate infinite sets of strategies. Subsection 4.1 implements an explicit and discrete non-determinism in sequential games and sketches the proof of non-deterministic Nash equilibrium existence that has been formalised in Coq. Subsection 4.2 gives examples.

\subsection{Formal Development}

The explicit and discrete non-determinism is implemented this way: game trees need not be changed. Strategy profiles need changing. In order to be as simple as possible, the non-determinism of a choice among some options will be represented by a non empty subset of the options. In this article, the possible choices were left and right before. Now they are left, right or both.

Inductive Choice : Set $:=l-r-b$.

The only change in the definition of strategy profiles comes from the change in the definition of choice:

Inductive NDS : Type :=

$-s L:$ PayoffF $\rightarrow$ NDS

$-s N:$ Agent $\rightarrow$ Choice $\rightarrow N D S \rightarrow N D S \rightarrow N D S$.

Before, any strategy profile would induce a payoff. Now a strategy profile yields a non empty set of possible payoffs to each agent. This "set" is represented by a list in Coq mainly because the axioms of 
set theory are not needed. The non-deterministic payoff function below expects a strategy profile and an agent as arguments. The keyword "struct" in the Coq code below means that the function is defined by structural recursion (on its argument $s$ ). The symbol ++ means list concatenation (set union).

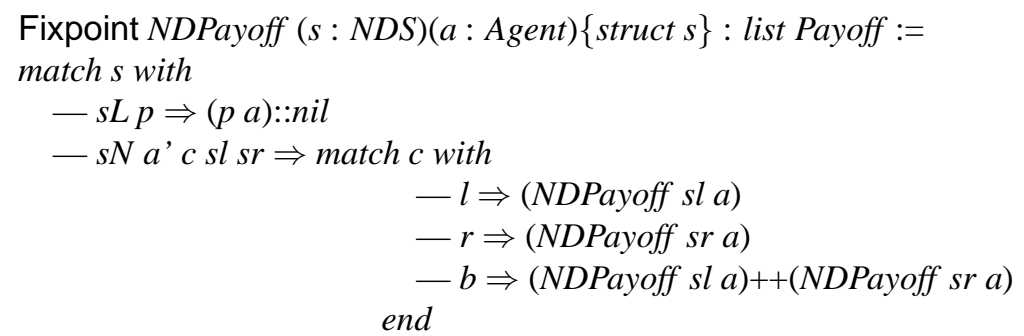

end.

In order to keep the essence of the definitions of Nash equilibrium and backward induction, two strategies need comparing through their induced set of possible outcomes. Here the preference relation is transitive and irreflexive. Transitivity reflects agents being consistent and aware at a global level. Irreflexivity means that nothing is strictly better than itself. Now we discuss the way to define a comparison among sets or lists. Informally, a set $A$ of outcomes is better than a set $B$ if whatever outcome nondeterministically chosen in $A$ and whatever outcome non-deterministically chosen in $B$, the one from $A$ is always better than or equivalent to the one from $B$, and sometimes it is strictly better. In the formal proof, I do not use set theory. The notion of list is enough and seems to be more practical: it uses fewer libraries, it is constructive, it does not require the extensionality axiom from set theory, and the inductive proof principle is always available. However I may sometimes use the word of set in an informal meaning.

In the following definition, AllLe $l l^{\prime}$ 'means that any element of the list $l$ is less than or equal to any element of the list $l$ ', w.r.t. the relation partially ordering the payoffs. And SomeLt $l l$ ' means that some element in $l$ is strictly less than some element in $l$ '. The predicate PrefL compares lists and the predicate PrefS compares strategy profiles.

Definition PrefL ( $\left(l^{\prime}\right.$ : list Payoff) : Prop := SomeLt l l' $\wedge$ AllLe l l'.

\section{Definition PrefS $(a$ : Agent $)(s 1$ s2 : NDS) : Prop :=} PrefL (NDPayoff s1 a)(NDPayoff $s 2 a)$.

The two independent following remarks are worth noting in order to get a intuition of the preference relation: first, if an element of a set and another element of another set are not comparable then the two sets should not be comparable. Second, the two sets $\{0,2\}$ and $\{3,1\}$, for example, are not comparable.

The agent-convertibility is the "natural" generalisation of the deterministic one. The definition of Nash equilibrium is conceptually the same as before:

Definition $N D E q(s: N D S):$ Prop :=

$$
\forall a, \forall s^{\prime}, \text { NDAgentConv a } s s^{\prime} \rightarrow \neg \text { PrefS a s s'. }
$$

It would be possible to define a backward induction predicate. The definition would change slightly to take the non-determinism into account, but the backward induction spirit would still be present. However, the example of Subsection 3.2 would still mean that non-deterministic backward induction does not imply non-deterministic Nash equilibrium. So, a proof of existence of non-deterministic Nash equilibrium 
cannot follow Vestergaard (2006). Instead I define a non-deterministic backward induction function and prove directly that it is a non-deterministic Nash equilibrium. Informally, the function is defined as follow: three cases can occur at a node: left is better than right, right is better than left, or left and right are not comparable (the case left better than right and right better than left is impossible by transitivity and irreflexivity). If one option is better than the other one then the agent chooses that better option. If the two options are not comparable then the agent non-deterministically chooses the two options via the keyword "both". For arbitrary (non binary) games, the agent non-deterministically chooses all the maximal options. In the inductive definition below, PrefS_dec means that PrefS is decidable and that a case splitting is performed. When reading the syntax as pseudo code, PrefS_dec can be informally replaced by Pref $S$ :

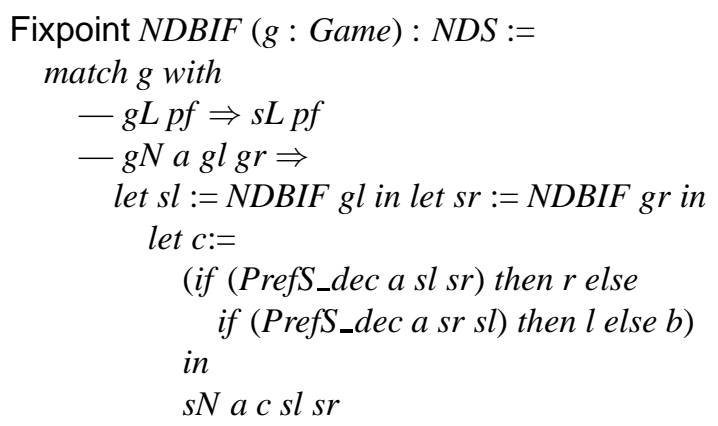

end.

Before stating the theorem I need to define a function that takes a strategy profile and retrieves the game the strategy profile is built on. It amounts to deleting the choices in a strategy profile.

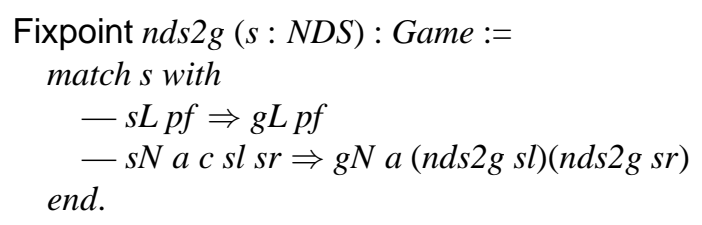

Now we can state the theorem:

Theorem Equilibrium_constructive_existence :

$\forall g:$ Game, $n d s 2 g(N D B I F g)=g \wedge N D E q(N D B I F g)$.

Both conjuncts are proved by induction. The first one and the leaf case of the second one are straightforward. The node case of the second requires a few lemmas on the preference relation PrefS. It proceeds by case splitting on that relation w.r.t. the two sub-strategy profiles. Three cases out of four need a subcase splitting on the choice. The most difficult case occurs when the two sub-strategy profiles are not comparable.

\subsection{Example}

In the following example, the sequential game involves two agents $a$ and $b$. The set of payoffs is the set of the finite subsets of the naturals. A singleton $\{n\}$ is represented by the number $n$ alone. At a leaf the label 
$A, B$ means that agent $a$ gets a payoff $A$ and that agent $b$ gets a payoff $B$. In a strategy profile, choices are represented by bold lines between nodes. Here is the non-deterministic backward induction.

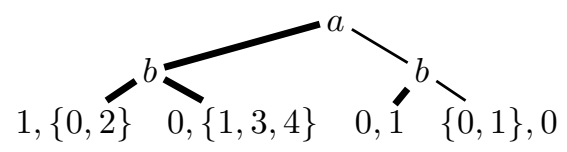

So, a play of the game according to the non-deterministic backward induction function will result in $a$ choosing left, then $b$ non-deterministically choosing left or right. In addition, the non-deterministic backward induction function may be of interest beyond its technical use in the proof. Compare the two games and non-deterministic backward inductions below:

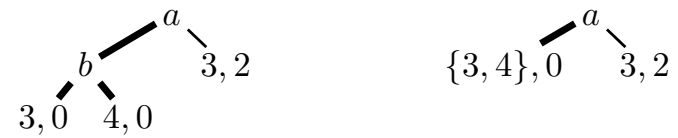

Informally, the game to the right is actually the way agent $a$ sees the game to the left in a noncooperative setting and when at the root of the game tree: if $a$ goes to the right he will get 3 . If he goes to the left he will get either 3 or 4 (but has no clue how $b$ is going to choose). This suggests that sequential games over partial orders are relevant even when dealing with traditional sequential games. In particular, the non-deterministic backward induction function may help recommend players how to play.

\section{Conclusion and future work}

Game theory usually considers real-valued payoffs. This article considers partially ordered payoff domains for sequential games. Game theory usually considers non-determinism as a consequence of probabilities. This article considers an explicit, i.e., direct and intensional, non-determinism. That nondeterminism is introduced to solve the issue raised by the partial ordering. That issue is: if the payoff domain is partially ordered, do Kuhn's result and its formal proof still hold? An example shows that with the traditional definition of Nash equilibrium, not all sequential games have Nash equilibria. Refining the definitions along Le Roux et al. (2006) is an improvement since there is no simple counter example to Nash equilibrium existence. However, it does not lead to an existence proof in the style of Vestergaard (2006). Indeed an example shows that backward induction does not imply Nash equilibrium, which is needed for that specific proof. Then, Section 4 introduces the formal notions of non-deterministic choice, strategy, and equilibrium. Those notions still permit examples where backward induction does not imply Nash equilibrium, therefore an equilibrium-existence result cannot be proved along the proof of Vestergaard (2006) in the partial-order/non-deterministic case. However, the setting allows the definition of a backward induction function that takes any game and returns a non-deterministic strategy which is both backward induction and Nash equilibrium for the game. This construction leads to a general adaptation of Kuhn's result keeping almost the same overall proof structure. This construction is an instance of what is called along Le Roux et al. (2006) a Nash-like construction. It is worth stressing that all the technical development was formalised with the proof assistant Coq.

This article can lead to at least three other research topics. 1) The spirit of backward induction having been kept to some extent, it is reasonable to wonder whether the results involving backward induction in traditional game theory can be easily generalised to the partial-order/non-deterministic case. 2) An example shows that even with a refined deterministic definition equilibrium, the proof of Kuhn's result 
does not hold. But does the result itself hold? Said otherwise: has any sequential game with partially ordered payoff domain a (deterministic) Nash equilibrium? 3) The explicit and discrete non-determinism has been successfully used for sequential games. Could it lead to a non-deterministic notion of strategy and a simple proof of equilibrium for strategic games?

\section{Acknowledgements}

I thank Pierre Lescanne and René Vestergaard for their comments, Damien Pous for sharing his knowledge in Coq, the first referee for pointing me to multi criteria games and the second referee for suggesting slight presentation changes.

\section{References}

D. Blackwell. An analog of the minimax theorem for vector payoffs. Pacific Journal of Mathematics, 6: $1-8,1956$.

H. W. Kuhn. Extensive games and the problem of information. Contributions to the Theory of Games II, 1953.

S. Le Roux, P. Lescanne, and R. Vestergaard. A discrete Nash theorem with low complexity and dynamic equilibria. Research Report IS-RR-2006-006, JAIST, May 2006.

J. Nash. Equilibrium points in n-person games. Proceedings of the National Academy of Sciences, 36: 48-49, 1950.

J. v. Neumann and O. Morgenstern. Theory of Games and Economic Behavior. Princeton Univ. Press, Princeton, 1944.

R. Selten. Spieltheoretische Behandlung eines Oligopolmodells mit Nachfrageträgheit. Zeitschrift für die gesamte Staatswissenschaft, 121, 1965.

R. Selten. Reexamination of the perfectness concept for equilibrium points in extensive games. International Journal of Game Theory, 4, 1975.

R. Vestergaard. A constructive approach to sequential Nash equilibria. Information Processing Letter, 97: 46-51, 2006.

E. Zermelo. Über eine Anwendung der Mengenlehre auf die Theorie des Schachspiels. Proceedings of the Fifth International Congress of Mathematicians, II, 1912. 
